Supplementary Information

\title{
Facile Synthesis of Monomeric Alumatranes
}

\author{
Weiping Su, Youngjo Kim, Arkady Ellern, Ilia A. Guzei and John G. Verkade* \\ Department of Chemistry, Iowa State University, Ames, IA 50011 \\ E-mail: jverkade@iastate.edu
}

1. Table of assignments of ${ }^{1} \mathrm{H}$ and ${ }^{13} \mathrm{C}$ NMR spectra for compound $\mathbf{2 - 5}$ and $\mathbf{7}$

2. ${ }^{1} \mathrm{H}$ and ${ }^{13} \mathrm{C}$ NMR spectra of compound $\mathbf{2 - 5}$ and 7 
Table of assignments of ${ }^{1} \mathrm{H}$ and ${ }^{13} \mathrm{C}$ NMR spectra for compound 2-5 and $\mathbf{7}^{\mathrm{a}}$

\begin{tabular}{|c|c|c|}
\hline Compound & ${ }^{1} \mathrm{H}$ NMR $(\delta)$ & ${ }^{13} \mathrm{C}$ NMR $(\delta)$ \\
\hline 2 & $\begin{array}{l}6.86(\mathrm{~s}, 3 \mathrm{H}, \mathrm{Ar}), 6.60(\mathrm{~s}, 3 \mathrm{H}, \mathrm{Ar}), 4.56(\mathrm{~b}, \\
\left.4 \mathrm{H}, \mathrm{CH}_{2} \mathrm{CH}_{2} \mathrm{O}\right), 4.25(\mathrm{~d}, \mathrm{~J}=10.2 \mathrm{~Hz}, 3 \mathrm{H}, \\
\left.\mathrm{ArCH}_{2} \mathrm{~N}\right), 2.83\left(\mathrm{~d}, \mathrm{~J}=10.2,3 \mathrm{H}, \mathrm{ArCH}_{2} \mathrm{~N}\right), \\
2.19-2.18 \quad\left(\mathrm{~m}, \quad 22 \mathrm{H}, \quad \mathrm{ArCH}_{3} \quad \text { and }\right. \\
\left.\mathrm{CH}_{2} \mathrm{CH}_{2} \mathrm{O}\right) .\end{array}$ & $\begin{array}{lll}154.5 \quad(\mathrm{Ar}) ; & 131.2(\mathrm{Ar}) ; 127.2 & (\mathrm{Ar}) ; \\
127.1(\mathrm{Ar}) ; 126.0(\mathrm{Ar}) ; 120.8(\mathrm{Ar}) ; & 71.6 \\
\left(\mathrm{CH}_{2} \mathrm{O}\right) ; \quad 58.9 \quad\left(\mathrm{ArCH} \mathrm{H}_{2} \mathrm{~N}\right) ; & 25.9 \\
\left(\mathrm{CH}_{2} \mathrm{CH}_{2} \mathrm{O}\right) ; 20.7 \quad\left(\mathrm{ArCH}_{3}\right) ; & 17.3 \\
\left(\mathrm{ArCH}_{3}\right) . & & \end{array}$ \\
\hline 3 & $\begin{array}{l}10.06(\mathrm{~s}, 1 \mathrm{H}, \mathrm{PhCHO}), 7.61(\mathrm{~d}, \mathrm{~J}=7.0 \mathrm{~Hz}, \\
2 \mathrm{H}, \operatorname{ArCHO}), 7.0(\mathrm{~s}, 3 \mathrm{H}, \mathrm{Ar}), 6.84-6.79 \\
(\mathrm{~m}, 3 \mathrm{H}, \mathrm{ArCHO}), 6.51(\mathrm{~s}, 3 \mathrm{H}, \mathrm{Ar}), 4.39(\mathrm{~b}, \\
\left.3 \mathrm{H}, \mathrm{ArCH}_{2} \mathrm{~N}\right), 2.75\left(\mathrm{~b}, 3 \mathrm{H}, \mathrm{ArCH}_{2} \mathrm{~N}\right), 2.34 \\
\left(\mathrm{~s}, 9 \mathrm{H}, \mathrm{ArCH}_{3}\right), 2.23\left(\mathrm{~s}, 9 \mathrm{H}, \mathrm{ArCH}_{3}\right) .\end{array}$ & 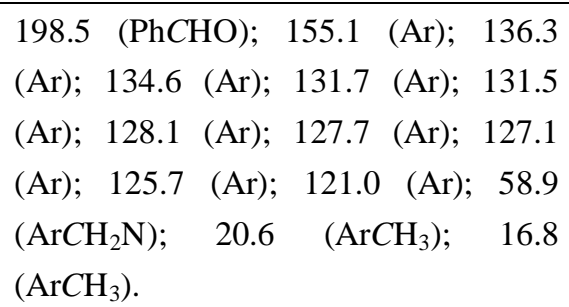 \\
\hline $4^{b}$ & $\begin{array}{l}6.97(\mathrm{~s}, 2 \mathrm{H}, \mathrm{Ar}) ; 6.90(\mathrm{~s}, 1 \mathrm{H}, \mathrm{Ar}) ; 6.47(\mathrm{~s}, \\
2 \mathrm{H}, \mathrm{Ar}) ; 6.43(\mathrm{~s}, 1 \mathrm{H}, \mathrm{Ar}) ; 4.26(\mathrm{~d}, \mathrm{~J}=14.2 \\
\left.\mathrm{Hz}, \quad 3 \mathrm{H}, \quad \mathrm{ArCH} \mathrm{H}_{2} \mathrm{~N}\right) ; 3.02 \quad(\mathrm{~b}, \quad 2 \mathrm{H}, \\
\left.\mathrm{AlNH}_{2} \mathrm{CH}_{2}\right) ; \quad 2.83 \quad(\mathrm{~m}, \quad 2 \mathrm{H}, \\
\left.\mathrm{AlNH}_{2} \mathrm{CH}_{2} \mathrm{CH}_{2} \mathrm{NH}_{2}\right) ; 2.46(\mathrm{~d}, \mathrm{~J}=14.0 \mathrm{~Hz}, \\
\left.3 \mathrm{H}, \mathrm{ArCH}_{2} \mathrm{~N}\right) ; 2.30\left(\mathrm{~s}, 9 \mathrm{H}, \mathrm{ArCH}_{3}\right) ; 2.23 \\
(\mathrm{~s}, 6 \mathrm{H}, \mathrm{ArCH}) ; 2.19\left(\mathrm{~s}, 3 \mathrm{H}, \mathrm{ArCH}_{3}\right), \\
2.09-2.05\left(\mathrm{~m}, 2 \mathrm{H}, \mathrm{NH}_{2}\right) .\end{array}$ & $\begin{array}{llll}155.1(\mathrm{Ar}) ; & 131.2 & (\mathrm{Ar}) ; 129.2 & (\mathrm{Ar}) ; \\
126.6(\mathrm{Ar}) ; & 125.5(\mathrm{Ar}) ; 121.1(\mathrm{Ar}) ; & 58.9 \\
\left(\mathrm{ArCH}_{2} \mathrm{~N}\right) ; & 43.6 & \left(\mathrm{NH}_{2} \mathrm{CH}_{2}\right) ; & 41.8 \\
\left(\mathrm{NH}_{2} \mathrm{CH}_{2}\right) ; & 20.6 & \left(\mathrm{ArCH}_{3}\right) ; & 17.0 \\
\left(\mathrm{ArCH}_{3}\right) . & & & \end{array}$ \\
\hline 5 & $\begin{array}{l}7.09(\mathrm{~m}, 5 \mathrm{H}, \mathrm{OPh}) ; 6.78(\mathrm{~s}, 3 \mathrm{H}, \mathrm{Ar}) ; 6.54 \\
(\mathrm{~s}, 3 \mathrm{H}, \mathrm{Ar}) ; 5.38\left(\mathrm{~d}, \mathrm{~J}_{\mathrm{PH}}=495.1 \mathrm{~Hz}, 1 \mathrm{H},\right. \\
\mathrm{PH}) ; 4.25\left(\mathrm{~b}, 3 \mathrm{H}, \mathrm{ArCH}_{2} \mathrm{~N}\right), 3.47-3.34(\mathrm{~m}, \\
\left.3 \mathrm{H}, \mathrm{NCHMe}_{2}\right) ; 3.08-3.06(\mathrm{~m}, 6 \mathrm{H}, \\
\left.\mathrm{CH}_{2} \mathrm{NCHMe}_{2}\right) ; 2.76-2.70(\mathrm{~m}, 9 \mathrm{H}, \\
\left.\mathrm{N}\left(\mathrm{CH}_{2}\right)_{3} \text { and } \mathrm{ArCH}_{2} \mathrm{~N}\right) ; 2.16(\mathrm{~s}, 9 \mathrm{H}, \\
\left.\mathrm{ArCH}_{3}\right) ; 2.07\left(\mathrm{~s}, 9 \mathrm{H}, \mathrm{ArCH}_{3}\right) ; 1.02(\mathrm{~d}, \mathrm{~J}= \\
\left.6.7 \mathrm{~Hz}, 18 \mathrm{H}, \mathrm{NCH}\left(\mathrm{CH}_{3}\right)_{2}\right) .\end{array}$ & 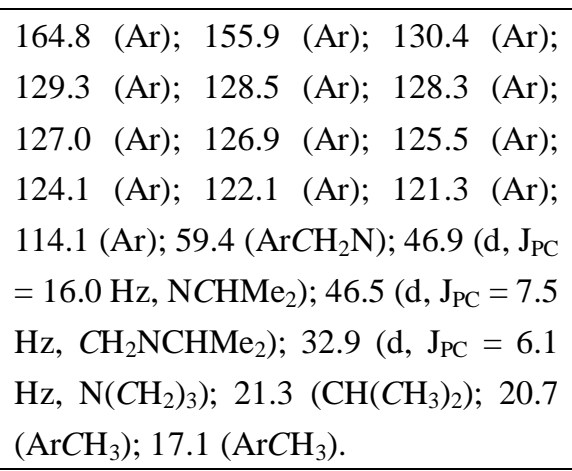 \\
\hline 7 & $\begin{array}{l}9.09(\mathrm{~b}, 2 \mathrm{H}, \mathrm{AlOH})_{2} ; 6.91(\mathrm{~s}, 1 \mathrm{H}, \mathrm{Ar}) \\
6.62-6.53(\mathrm{~m}, 3 \mathrm{H}, \mathrm{Ar}) ; 6.35(\mathrm{~s}, 1 \mathrm{H}, \mathrm{Ar}) \\
5.96(\mathrm{~s}, 1 \mathrm{H}, \mathrm{Ar}) ; 4.35(\mathrm{~d}, \mathrm{~J}=13.8 \mathrm{~Hz}, 1 \mathrm{H}, \\
\left.\mathrm{ArCH}_{2} \mathrm{~N}\right) ; 4.16-4.04\left(\mathrm{~m}, 2 \mathrm{H}, \mathrm{ArCH} \mathrm{A}_{2} \mathrm{~N}\right) ; \\
3.07(\mathrm{~s}, 1 \mathrm{H}, \mathrm{OH}) ; 2.81-2.67(\mathrm{~m}, 3 \mathrm{H}, \\
\left.\mathrm{ArCH}_{2} \mathrm{~N}\right) ; 2.32\left(\mathrm{~s}, 3 \mathrm{H}, \mathrm{ArCH}_{3}\right) ; 2.25(\mathrm{~s}, 3 \\
\left.\mathrm{H}, \mathrm{ArCH}_{3}\right) ; 2.08\left(\mathrm{~s}, 3 \mathrm{H}, \mathrm{ArCH}_{3}\right) ; 1.96(\mathrm{~s}, \\
\left.3 \mathrm{H}, \mathrm{ArCH}_{3}\right) ; 1.71\left(\mathrm{~s}, 3 \mathrm{H}, \mathrm{ArCH}_{3}\right) ; 1.67 \\
\left(\mathrm{~s}, 3 \mathrm{H}, \mathrm{ArCH}_{3}\right) .\end{array}$ & $\begin{array}{l}153.9(\mathrm{Ar}) ; 153.4(\mathrm{Ar}) ; 152.7(\mathrm{Ar}) ; \\
132.6(\mathrm{Ar}) ; 131.6(\mathrm{Ar}) ; 130.3(\mathrm{Ar}) ; \\
127.7(\mathrm{Ar}) ; 127.4(\mathrm{Ar}) ; 127.2(\mathrm{Ar}) ; \\
125.6(\mathrm{Ar}) ; 125.1(\mathrm{Ar}) ; 121.9(\mathrm{Ar}) ; \\
121.8(\mathrm{Ar}) ; 118.2(\mathrm{Ar}) ; 59.4\left(\mathrm{ArCH} \mathrm{H}_{2} \mathrm{~N}\right) ; \\
58.7\left(\mathrm{ArCH} \mathrm{H}_{2} \mathrm{~N}\right) ; 20.8\left(\mathrm{ArCH}_{3}\right) ; 20.6 \\
\left(\mathrm{ArCH}_{3}\right) ; 20.3\left(\mathrm{ArCH}_{3}\right) ; 17.8\left(\mathrm{ArCH}_{3}\right) ; \\
16.9\left(\mathrm{ArCH}_{3}\right) ; 15.7\left(\mathrm{ArCH}_{3}\right) .\end{array}$ \\
\hline
\end{tabular}

${ }^{\text {a } 1} \mathrm{H}$ NMR spectra and ${ }^{13} \mathrm{C}$ NMR spectra were recorded on a Varian Germini-300 spectrometer at $300 \mathrm{M} \mathrm{Hz}$ and $75.5 \mathrm{M} \mathrm{Hz}$, respectively. $\mathrm{CD}_{2} \mathrm{Cl}_{2}$ was used as a solvent. ${ }^{\mathrm{b}} \mathrm{C}_{6} \mathrm{D}_{6}$ was used as a solvent. 


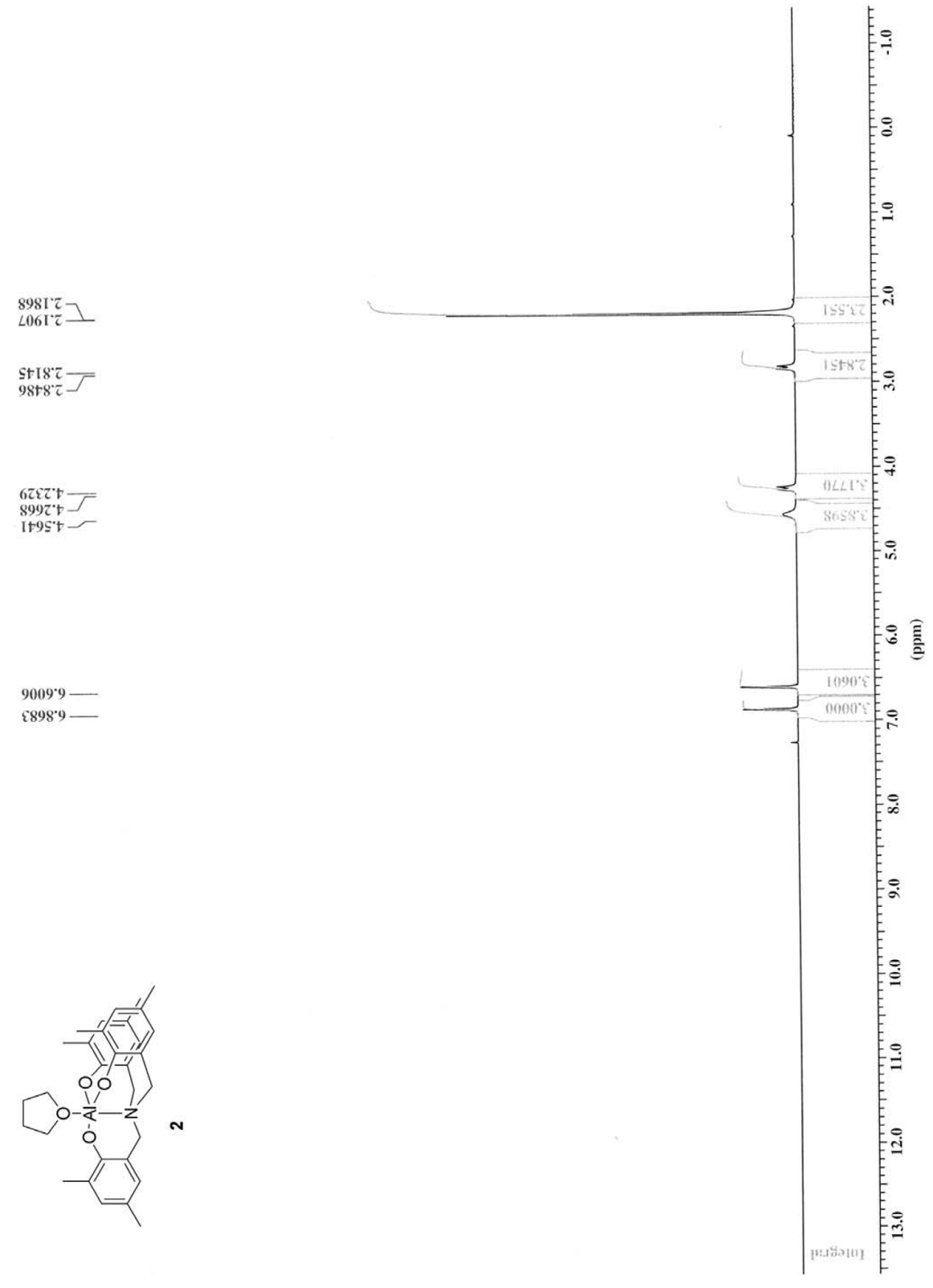




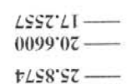

tt88'ss -

teco $1 L$

9z88.0Z1 -

9LE0.

$\varsigma z 8[\cdot \angle \tau]]$

86I TEI

tLLt*tSI -
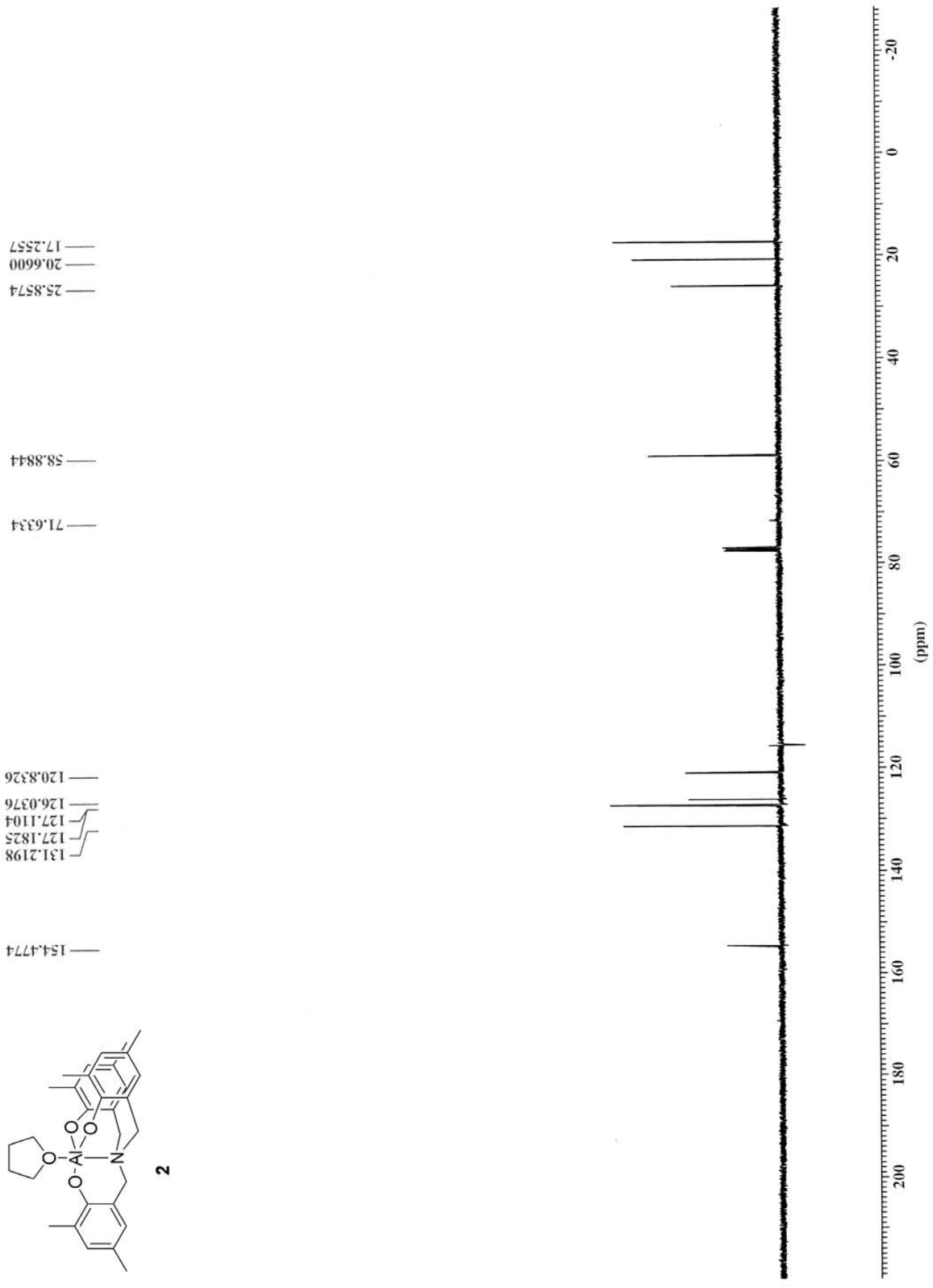

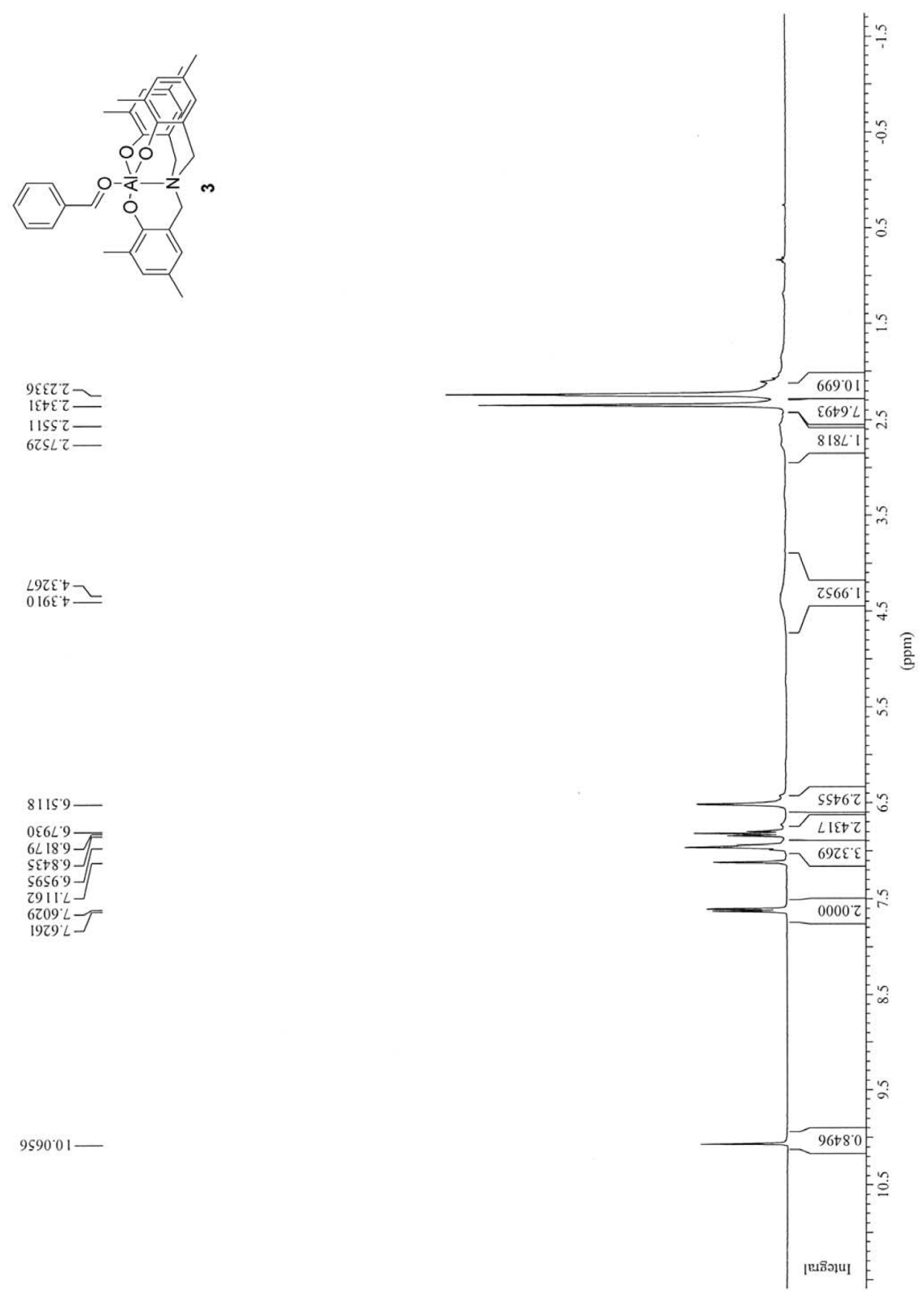


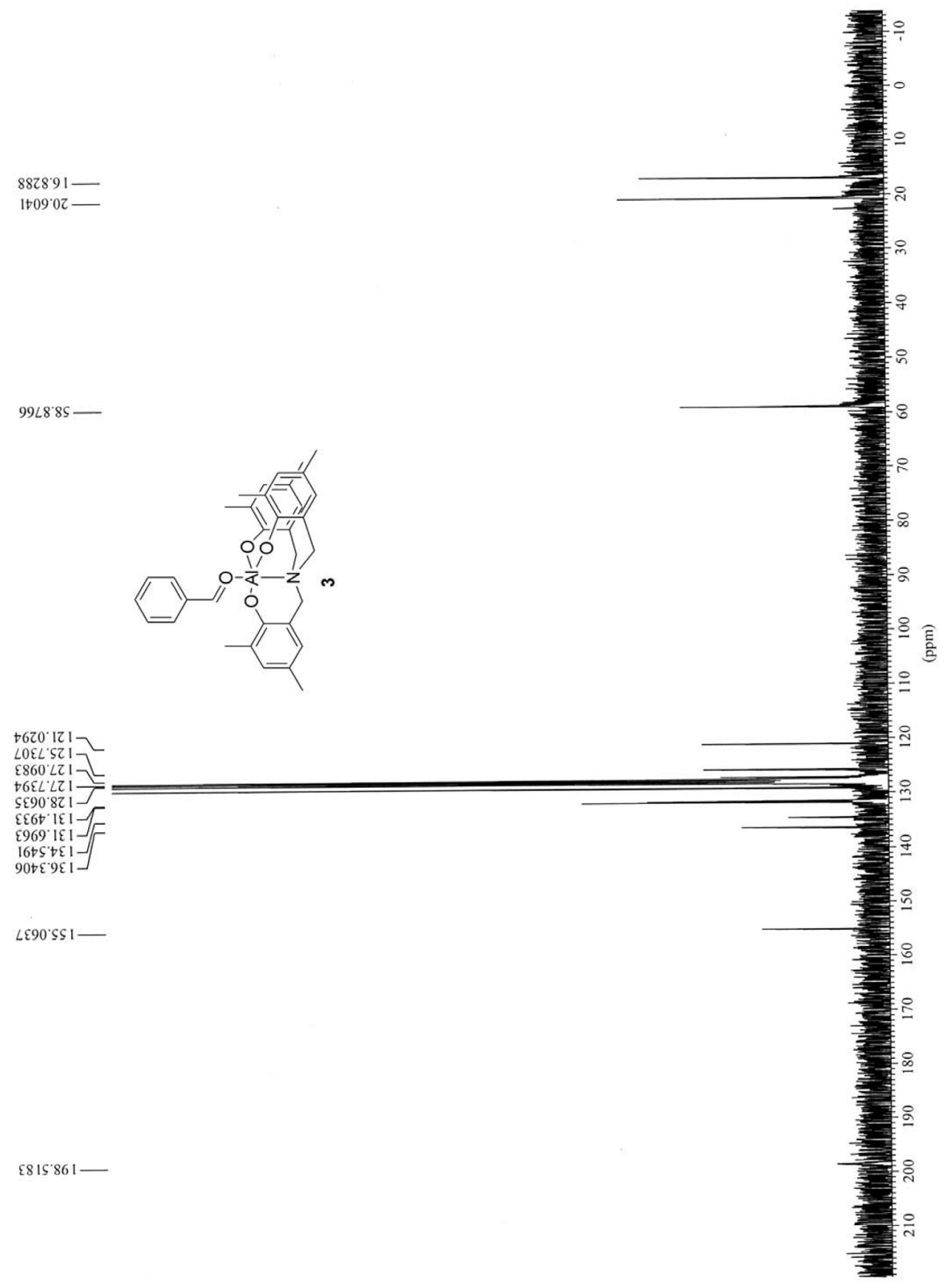




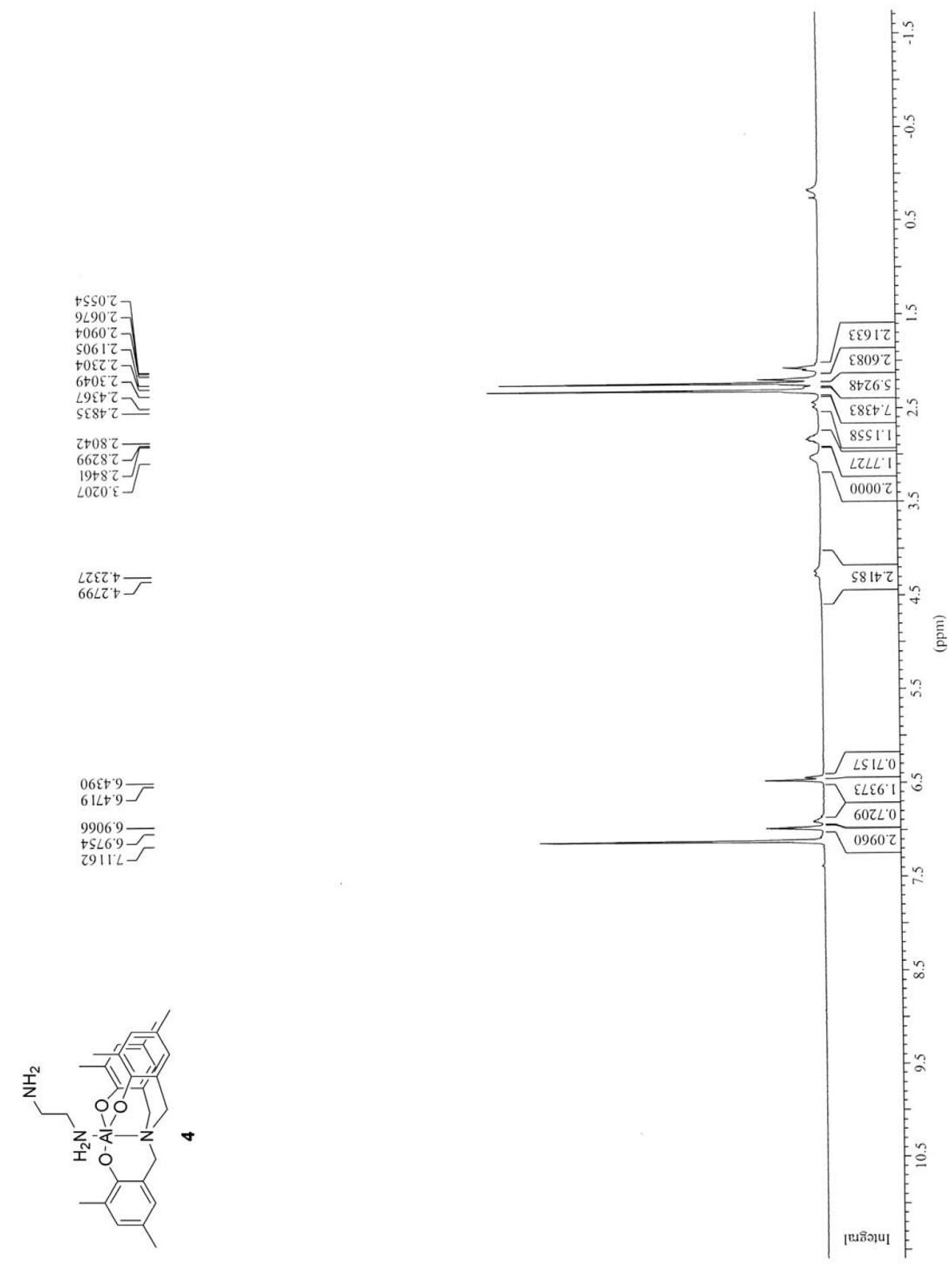




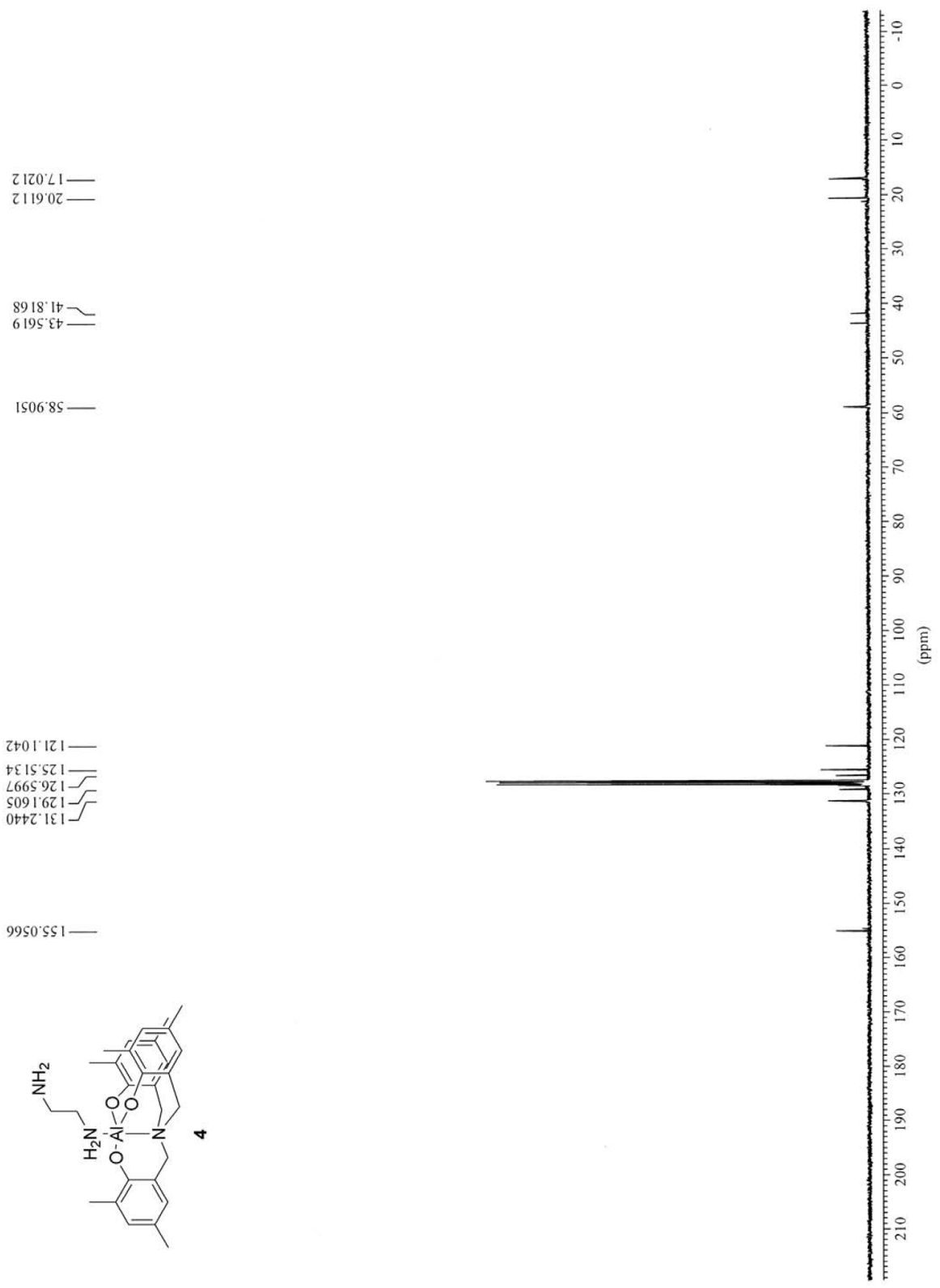



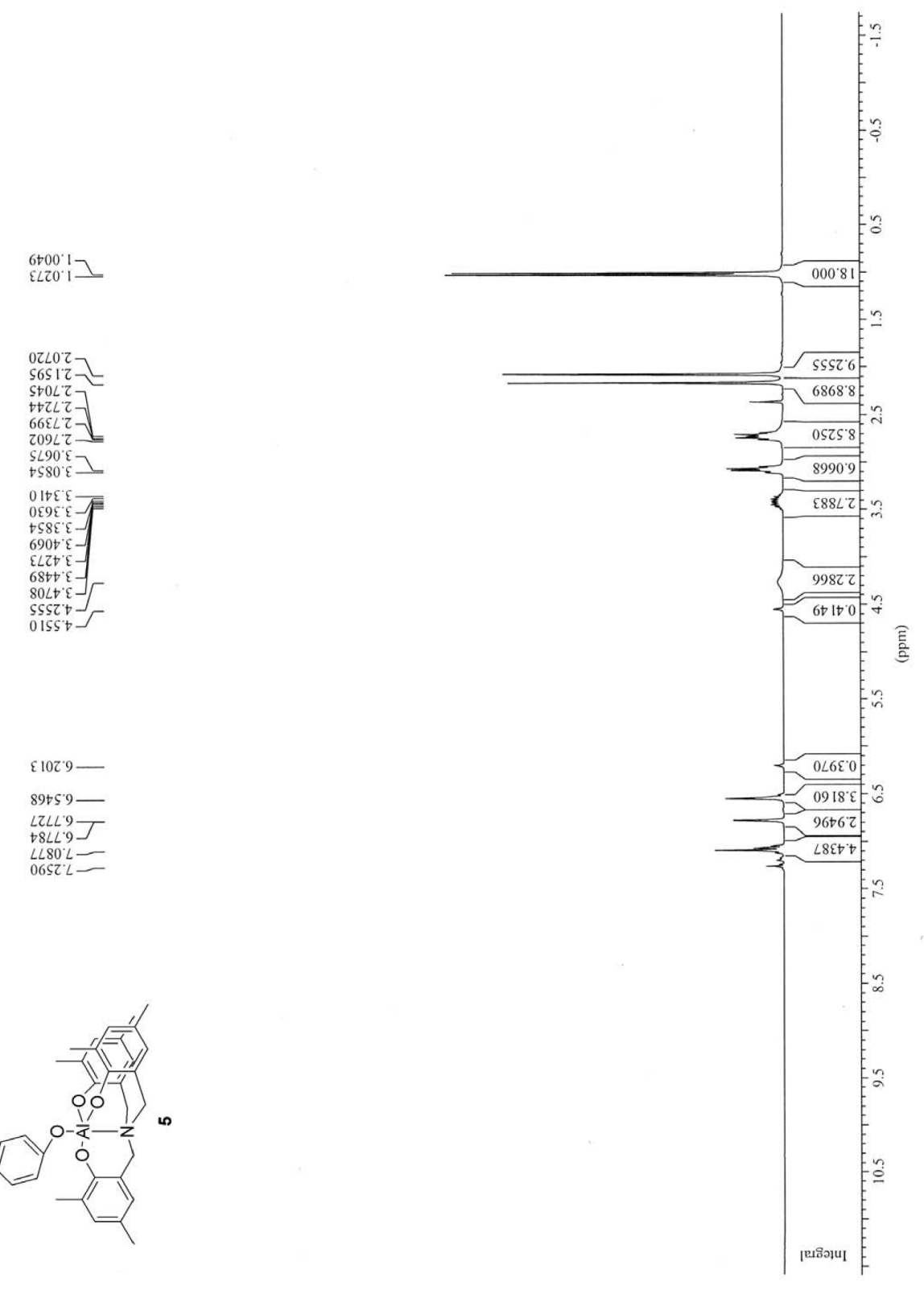

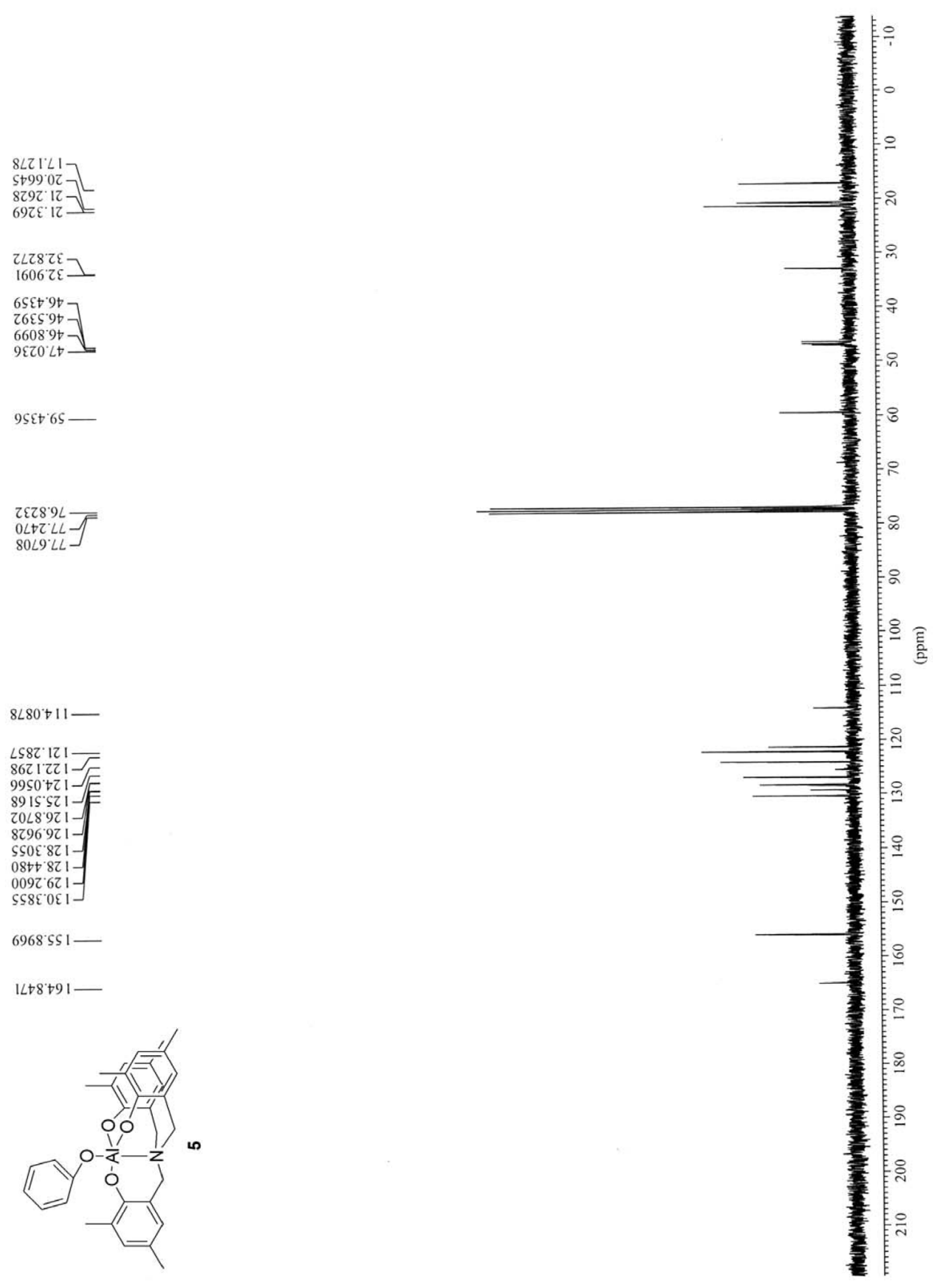

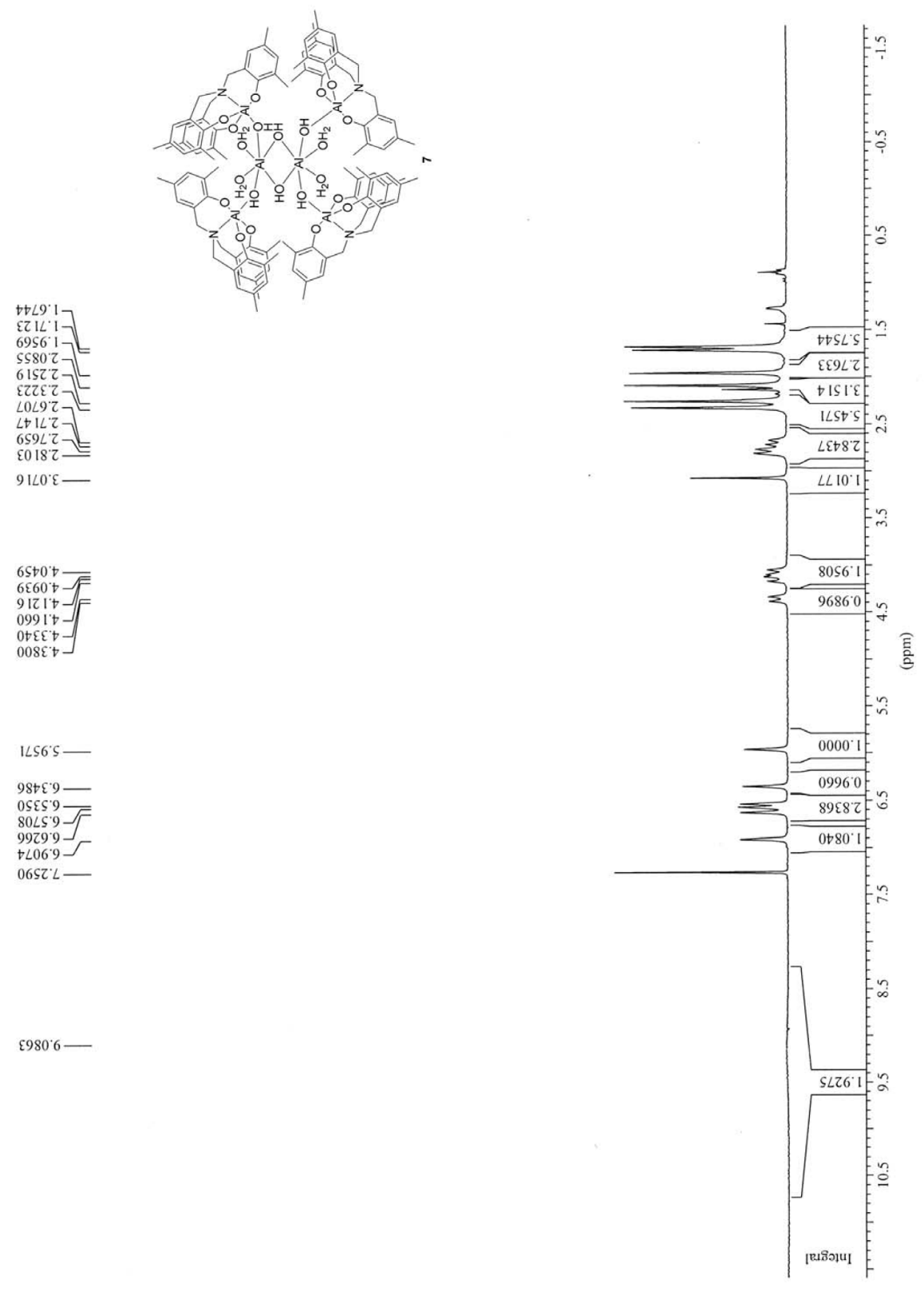
E8L9' $\$ I

$2+1691$

TESLLI

06 IE $0 Z$

$\left.\begin{array}{l}\angle 179^{\circ} 02 \\ 1684^{\circ} 07\end{array}\right]$ E

toeL $8 S$ 乙

66228117

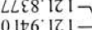

$0176.121-$

$1029^{\circ} \mathrm{SZl} 1$

$\langle S| Z \angle Z I-]=E$

$\angle S E L L Z I$ I

$6 S \zeta 18 Z 1-$

c28c0el-

LZ†9 IEI-

6SS9'ZSI-

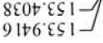

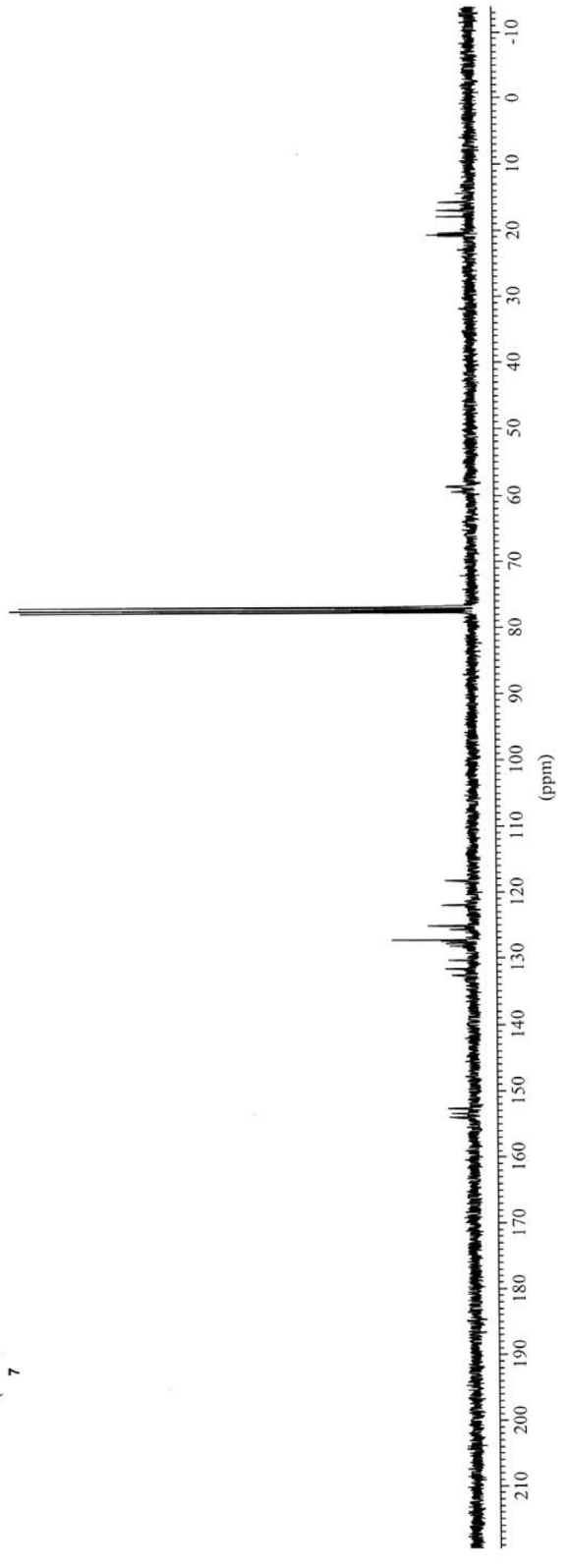

12 